\title{
Modelo para setorizar redes de distribuição de água baseado nas características das unidades consumidoras
}

\author{
Marcele Elisa Fontana ${ }^{a *}$, Danielle Costa Morais ${ }^{b}$ \\ a*marcelelisa@gmail.com, UFPE, Brasil \\ bdaniellemorais@yahoo.com.br, UFPE, Brasil
}

\begin{abstract}
Resumo
Além dos custos diretos, a ocorrência de rupturas na rede de distribuição de água tem sempre consequências sociais associadas, principalmente pela interrupção do abastecimento de água durante a reparação da infraestrutura. Nesses casos, a setorização da rede torna mais fácil qualquer atividade de manutenção. Porém, além do elevado custo da alocação de válvulas de fechamento, as normas brasileiras sobre setorização das redes dizem respeito apenas a questões técnicas que devem ser obedecidas. Portanto, propõe-se uma setorização que também incorpore a intensidade do impacto gerado pela falta de água em cada trecho da tubulação. Para medir esse impacto é proposto um índice de priorização que agrega as características das unidades consumidoras por meio do método multicritério SMARTER. Assim, desenvolve-se um modelo de setorização para manobra que leva em consideração esse índice, buscando a maximização dos benefícios gerados e a minimização do número de válvulas de fechamento.

Palavras-chave

Manutenção de redes de distribuição de água. Setor de manobra. Válvulas de fechamento. Características das unidades consumidoras. SMARTER.
\end{abstract}

\section{Introdução}

O objetivo principal de todos os sistemas de distribuição de água é o fornecimento de água para atender à demanda. No entanto, um dos principais desafios para os gestores de abastecimento de água é a minimização das perdas de água causadas por vazamentos, principalmente decorrentes de problemas de uma infraestrutura envelhecida. 0 controle de perdas tem se tornado de grande interesse mundial devido a crescente tendência internacional para a sustentabilidade, a eficiência econômica e a proteção do ambiente. No âmbito desse objetivo, altos investimentos são realizados anualmente em detecção e reparo de vazamentos (CHRISTODOULOU; DELIGIANNI, 2010; DELGADO-GALVÁN et al., 2010; MORAIS; ALMEIDA, 2006; KIM; MAYS, 1994; LAMBERT; HIRNER, 2002; MOUNCE; BOXALL; MACHELL, 2010; TROJAN; MORAIS, 2012a).

Em geral, problemas de abastecimento de água envolvem a sociedade como um todo, não só por se tratar de um recurso escasso, mas pelo grau de importância que tem na vida das pessoas. Por esse fato, decisões que envolvem a rede de distribuição de água têm um caráter social. Dessa forma, segundo Morais e Almeida (2007), é necessário pesquisar as possíveis soluções para minimizar os problemas de uma manutenção das redes inadequada através de uma estratégia específica de gestão de perdas. lsso porque, além dos motivos acima e dos custos diretos associados, conforme afirmação de Ramos et al. (2001), a ocorrência de vazamentos na rede pode levar à redução da água disponível e até à interrupção do abastecimento de água durante a reparação da infraestrutura, gerando a insatisfação dos usuários.

Nas redes de distribuição de água, as válvulas de isolamento ou fechamento são de grande interesse porque determinam a extensão do isolamento necessário para inspeção, reparação ou manutenção de parte do sistema (JUN et al., 2007; OZGER; MAYS, 2004). Nesse sentido, quanto menor for a região isolada (setor de manobra) para a realização 
de uma manutenção, menor será a quantidade de unidades consumidoras afetadas pela interrupção do fornecimento de água.

A alocação de válvulas eleva substancialmente os custos das empresas abastecedoras de água, tanto pelo custo da implantação quanto pela sua manutenção. Além disso, as normas brasileiras que regulamentam a setorização das redes de distribuição de água no Brasil são insuficientes para a determinação ideal dos setores, tendo em vista que não observam as características das unidades consumidoras. Por exemplo, a interrupção do abastecimento de água em um hospital não deveria ser encarada do mesmo modo que o desabastecimento de duas ou três residências. Na prática verifica-se uma setorização, de certa forma, intuitiva por parte dos técnicos.

Assim, verifica-se a necessidade de minimizar o uso de válvulas de fechamento e, ao mesmo tempo, garantir um nível de desempenho, ou benefício, adequado à população com a setorização da rede. Esse benefício pode ser medido pelo número de setores gerados, pois, como mencionado anteriormente, quanto mais setorizada for uma rede de distribuição de água, menos usuários serão impactados durante a interrupção do abastecimento. Contudo, sem o auxílio de um modelo específico, determinar esse número mínimo de válvulas e sua localização na rede pode ser uma tarefa difícil, principalmente em casos de redes de distribuição extensas.

Dessa forma, o objetivo deste trabalho é desenvolver um modelo de setorização para manobras que seja capaz de minimizar o número de válvulas de fechamento e, ao mesmo tempo, maximizar os benefícios gerados aos usuários pela setorização. Para tanto, uma etapa deste modelo é a proposta de um índice baseado nas características socioeconômicas das unidades consumidoras, com o intuito de medir a intensidade do impacto da falta de água em cada trecho de tubulação e, assim, auxiliar na tomada de decisões sobre a localização ideal das válvulas de fechamento, com a utilização mínima possível delas. Para a geração desse índice foi utilizado um método de agregação multicritério, o SMARTER.

Este artigo está seccionado da seguinte maneira. Na seção 2 são apresentados conceitos relativos à setorização para manobras. A seção 3 discorre sobre os métodos multicritério de apoio à decisão. $\mathrm{Na}$ seção 4 faz-se a proposição do índice de priorização dos trechos de tubulação. Em seguida, o modelo de setorização para manobras é definido e uma ilustração da aplicação é feita. Na seção 6 algumas discussões acerca do trabalho são feitas e, por fim, as considerações finais são realizadas na seção 7 .

\section{Setorização para manobras de rede de distribuição}

0 combate a perdas ou desperdícios implica a redução do volume de água não contabilizado, exigindo a adoção de medidas que permitam reduzir as perdas físicas e não físicas e mantê-las permanentemente em nível adequado, considerando a viabilidade técnico-econômica das ações de combate a perdas em relação ao processo operacional de todo o sistema (TROJAN; MORAIS, 2012b).

Nesse sentido, interrupções programadas (manutenção preventiva, por exemplo) e não planejadas (por exemplo, manutenção corretiva) ocorrem regularmente em redes de distribuição de água, tornando-se necessário isolar trechos de tubulações (MORAIS; CAVALCANTE; ALMEIDA, 2010; GIUSTOLISI; SAVIC, 2010). Em geral, a rede pode ser dividida em diferentes subsistemas, cada um pode representar condições de tubulação e características de consumo de água semelhantes (WU; SAGE; TURTLE, 2010). Nessa visão, levanta-se a problemática de setorização das redes de distribuição para a realização de manutenção necessária, tanto para prevenir perdas quanto para eliminá-las após sua detecção.

Conforme a NBR 12218/94 (ASSOCIAÇÃO..., 1994), um setor de manobras é a menor divisão da rede de distribuição cujo abastecimento pode ser isolado sem afetar o abastecimento do restante da rede. Esse setor deve abranger uma área que apresente uma ou mais das seguintes características: (a) Extensão de rede $=7$ mil a 35 mil metros $(m)$; (b) Número de economias $=600$ a 3 mil unidades; (c) Área $=40$ mil a 200 mil metros quadrados $\left(\mathrm{m}^{2}\right)$. Além disso, o isolamento do setor deve ser feito com o menor número de válvulas de fechamento.

As válvulas de fechamento, tal como o seu nome indica, são colocadas para isolar uma parte do sistema de distribuição de água para reparação, inspeção, ou manutenção (JUN, 2005; MAYS et al., 2000). Elas são normalmente localizadas em torno de junções desse sistema (OZGER; MAYS, 2004).

Segundo Ysusi (2000), a prática preferida para determinar a localização das válvulas é a instalação de uma válvula a menos do que o número de tubos (condutas) em uma junção, a chamada regra de válvula $(\mathrm{N}-1)$. Um sistema ideal exigirá duas válvulas para cada tubo, localizadas em suas extremidades, esse modelo é chamado de regra de válvula N. Já a norma NBR 12218/94 (ASSOCIAÇÃO..., 1994) diz que nas condutas secundárias deve ser prevista uma válvula junto ao ponto de ligação às condutas principais. Contudo, Heller e Pádua (2006) argumentam que essas regras e normas podem demasiar a utilização de válvulas em redes de distribuição. 
Portanto, a setorização para manobras em redes de distribuição de água apresenta um difícil trade-off entre os custos envolvidos, principalmente na implantação e manutenção das válvulas, e os benefícios gerados aos usuários com a setorização da rede.

\section{Apoio multicritério à decisão}

A grande vantagem da abordagem multiobjetivo reside no fato de que, de uma só vez, vários trade-offs entre as alternativas são encontrados, proporcionando assim um conjunto de soluções ótimas, com um nível diferente de compromisso entre os objetivos conflitantes (NICOLINI; ZOVATTO, 2009).

A escolha do método vai depender de vários fatores chave, tais como: o tempo disponível; o esforço requerido por uma dada abordagem; o conhecimento sobre o ambiente; a importância de uma decisão mais precisa; a necessidade de justificar a decisão para outros; o desejo de minimizar conflitos. Além desses, podem-se destacar outros fatores, tais como: o problema analisado; o contexto considerado; as informações disponíveis e o seu grau de precisão; a racionalidade requerida (compensatória ou não compensatória); a estrutura de preferências do decisor (DM) e a problemática do problema (ALMEIDA, 2011; ALMEIDA; COSTA, 2003).

Nesse sentido, há basicamente quatro diferentes problemáticas ou abordagens de problemas em que se podem usar métodos de apoio multicritério a decisão (BELTON; STEWART, 2002; ROY, 1996): Problemática

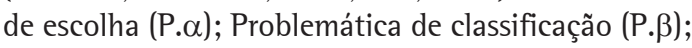
Problemática de ordenação (P. .); e Problemática de descrição (P.ঠ).

Almeida (2011) apresenta uma interessante classificação dos métodos que trabalham a problemática de ordenação: métodos ordinais, métodos de sobreclassificação e métodos de agregação aditivos.

Os métodos ordinais são típicos modelos para escolhas sociais. A função de escolha social é uma regra que atribui a cada conjunto de preferências individuais e alternativas um subconjunto de alternativas viáveis (NURMI, 1983). Destacam-se os métodos: lexicográfico, Borda, Condorcet e Copeland (BLACK, 1958; KLAMLER, 2005; NURMI, 1983; YOUNG, 1988).

Nos métodos de sobreclassificação as preferências do decisor são modeladas utilizando-se relações binárias de superação, $S$, que significa "pelo menos tão bom quanto” (FIGUEIRA; MOUSSEAU; ROY, 2006). Nessa abordagem encontram-se os métodos das famílias ELECTRE (Elimination and Choice Expressing Reality) (ROY; BERTIER, 1973; VINCKE, 1992) e da família PROMETHEE (Preference Ranking Organization Method for Enrichment Evaluations) (BELTON; STEWART, 2002).
Com relação aos métodos de agregação aditivos, eles consideram uma função valor $v_{j}(a)$ para cada critério $j$ para a obtenção da função valor global v(a) (ALMEIDA, 2011), ou seja, o valor global da alternativa a. Destacam-se os métodos: AHP (Analytic Hierarchy Process) (SAATY 1980), MACBETH (Measuring Attractiveness by a Categorical Based Evaluation Technique) (BANA E COSTA; VANSNICK, 1994), SMARTS (Simple Multi-Attribute Rating Technique) e SMARTER (Simple Multi-Attribute Rating Technique Extended to Ranking) (EDWARDS; BARRON, 1994).

\section{Proposta de um índice de priorização}

Para realizar a manutenção da rede de distribuição de água, na maioria das vezes é necessário interromper momentaneamente o fornecimento de água. Assim, verificou-se a necessidade de propor um índice de priorização dos trechos de tubulação que refletisse o quão indesejável é a interrupção do abastecimento em cada um deles. Para a geração do índice, as características levantadas (apresentadas na sequência) de cada trecho de tubulação devem ser agregadas em um único valor. Os métodos de agregação multicritérios são ideais para agregar essas avaliações que consideram cada característica como um critério de avaliação do trecho de tubulação.

\subsection{Levantamento das alternativas e critérios}

Para a geração do índice de priorização, as alternativas serão todos os trechos de tubulação pertencentes à rede de distribuição de água analisada. Os critérios serão algumas características relevantes das unidades consumidoras (economias). Neste trabalho, quatro características foram levantadas: número de economias; tipo de setor de cada economia; valor da tarifa paga por cada economia; e taxa de consumo médio da economia.

- $\mathrm{Cr}_{1}$ - Número de economias: Mede o número de unidades consumidoras em cada trecho de tubulação. Quanto maior o número de economias em um trecho, maior deve ser sua valoração no índice de prioridade, ou seja, menor deve ser o setor de manobra no qual se encontra esse trecho.

- $\mathrm{Cr}_{2}$ - Tipo de setor: Examina o tipo de estabelecimento existente na conduta. Os setores são classificados neste trabalho de acordo com a Tabela 1. Essa classificação é baseada na categorização cedida por uma empresa brasileira de distribuição de água. A ordem de importância de cada setor muda dependendo das características de cada município analisado. Essa categorização tem como base a economia de uma cidade costeira, onde o turismo, 
por exemplo, tem grade relevância. Em cada trecho de tubulação considera-se a classificação mais alta encontrada. Quanto maior a classificação do setor da economia, menor deve ser o setor para manobra. lsso se justifica, pois os impactos gerados pela falta de água em uma residência são consideravelmente menores do que em um hospital, por exemplo.

- $\mathrm{Cr}_{3}$ - Valor da tarifa: Montante total pago pelos usuários sobre a água utilizada. Para determinar o valor em cada trecho de tubulação é utilizado o consumo médio de água de cada estabelecimento constituinte desse trecho. Neste critério, quanto maior o valor, pior são as consequências da falta de água (do ponto de vista do fornecedor). A Tabela 2 apresenta as classes de tributação (em reais - $\mathrm{R} \$ / \mathrm{m}^{3}$ ), cedidas por uma empresa brasileira de distribuição de água.

Neste caso, quando uma unidade consumidora utiliza uma quantidade de água $x \leq 10 \mathrm{~m}^{3}$, o valor pago é único, ou seja, a taxa mínima. Após essa taxa é acrescido um valor a cada $\mathrm{m}^{3}$ de água consumida e esse valor também varia conforme a faixa de consumo. Por exemplo, se uma residência com tarifação normal consumir $15 \mathrm{~m}^{3}$ no mês, o valor cobrado será a taxa mínima de $\mathrm{R} \$ 24,52$ (referente a $10 \mathrm{~m}^{3}$ ) mais o excedente (referente a $5 \mathrm{~m}^{3}$ ), localizado na primeira faixa de consumo, que será de 5 vezes $\mathrm{R} \$ 2,81$, totalizando $\mathrm{R} \$ 38,57$.

- $\mathrm{Cr}_{4}$ - Consumo: Quantidade total média de água consumida na conduta. Quanto maior o consumo de água, maior é a insatisfação com a falta de água durante a interrupção do fornecimento e, portanto, menor deve ser o setor de manobra no qual se encontra esse trecho.

Embora o valor da tarifa $\left(\mathrm{Cr}_{3}\right)$ e o consumo de água $\left(\mathrm{Cr}_{4}\right)$ possam estar correlacionados, neste artigo serão tratados como independentes, uma vez que as informações que se deseja não estão diretamente relacionadas. Sobre o valor da tarifa deseja-se saber qual o montante monetário que a empresa arrecada naquele trecho em análise. Por ser uma empresa capitalista, ela vai priorizar o não desabastecimento de áreas onde a receita é maior. Em relação ao consumo deseja-se saber o volume de água consumido no trecho, pois quanto maior o volume, maiores são as chances de faltar água nos estabelecimentos durante uma manutenção.

Tabela 1. Classificação dos setores.

\begin{tabular}{|c|c|c|c|}
\hline Setor & Descrição & Setor & Descrição \\
\hline (1) Tarifas sociais & $\begin{array}{l}\text { 1.1-Residências } \\
\text { 1.2-Lote vago } \\
\text { 1.3-Hidrantes } \\
\text { 1.4-Parques e praças } \\
\text { 1.5-Templos, igrejas e instituições religiosas } \\
\text { 1.6-Condomínios }\end{array}$ & $\begin{array}{l}\text { (2) Serviços } \\
\text { públicos } \\
\text { ou sem fins } \\
\text { lucrativos }\end{array}$ & $\begin{array}{l}\text { 2.1-Entidade cuja mantenedora seja o Estado } \\
\text { 2.2-Organizações cívicas, políticas } \\
\text { 2.3-Entidade de classe e sindicais } \\
\text { 2.4-Entidades assistenciais } \\
\text { 2.5-Demais atividades de utilidade pública }\end{array}$ \\
\hline (3) Comércio & $\begin{array}{l}\text { 3.1-Instituições financeiras } \\
\text { 3.2-Serviços, lojas e escritórios } \\
\text { 3.3-Demais atividades comerciais } \\
\text { 3.4-Micro/pequeno comércio }\end{array}$ & (4) Residencial & $\begin{array}{l}\text { 4.1-Prédio ou residência sem piscina } \\
\text { 4.2-Prédio ou residência com piscina } \\
\text { 4.3-Conjuntos habitacionais }\end{array}$ \\
\hline (5) Outros serviços & $\begin{array}{l}\text { 5.1-Posto de gasolina com lavagem } \\
\text { 5.2-Bar/restaurante/supermercado/ lanchonete } \\
\text { 5.3-Escolas particulares } \\
\text { 5.4-Escolas públicas }\end{array}$ & & $\begin{array}{l}\text { 7.1-Construções para fins residenciais } \\
\text { 7.2-Construções para fins comerciais } \\
\text { 7.3-Beneficiamento de madeira/ serrarias/laminações } \\
\text { 7.4-Indústria de bebidas e fábricas de gelo }\end{array}$ \\
\hline (6) Turismo & $\begin{array}{l}\text { 6.1-Hotéis, pensões, motéis, saunas, similares } \\
6.2 \text {-Clubes e recreações }\end{array}$ & (7) Industrial & $\begin{array}{l}\text { 7.5-Construções para fins industriais } \\
\text { 7.6-Indústrias e fábricas (eletrônicas/mecânicas/ } \\
\text { metalúrgicas) }\end{array}$ \\
\hline $\begin{array}{l}\text { (8) Serviços de } \\
\text { saúde }\end{array}$ & $\begin{array}{l}\text { 8.1-Hospitais particulares } \\
8.2 \text {-Hospitais públicos }\end{array}$ & & $\begin{array}{l}\text { 7.7-Frigorificos/abatedouros/matadouros } \\
\text { 7.8-Indústrias química/farmacêutica/de velas/de papel } \\
\text { 7.9-Demais atividades industriais }\end{array}$ \\
\hline
\end{tabular}

Tabela 2. Tarifação $\left(\mathrm{R} \$ / \mathrm{m}^{3}\right)$ por mês.

\begin{tabular}{|c|c|c|c|c|c|c|}
\hline \multirow{2}{*}{ Consumo } & \multirow{2}{*}{ Taxa mínima até $10 \mathrm{~m}^{3}$} & \multicolumn{5}{|c|}{ Superior a $10 \mathrm{~m}^{3}$} \\
\hline & & $10<x \leq 20$ & $20<x \leq 30$ & $30<x \leq 50$ & $50<x \leq 90$ & $x \geq 90$ \\
\hline \multicolumn{7}{|l|}{ Residencial } \\
\hline Social & 5,25 & \multirow{2}{*}{$+2,81$} & \multirow{2}{*}{$+3,35$} & \multirow{2}{*}{$+4,60$} & \multirow{2}{*}{$+5,46$} & \multirow{2}{*}{$+10,48$} \\
\hline Normal & 24,52 & & & & & \\
\hline Comercial & 36,07 & $+7,15$ & $+7,15$ & $+7,15$ & $+7,15$ & $+7,15$ \\
\hline Industrial & 45,20 & $+9,58$ & $+9,58$ & $+9,58$ & $+9,58$ & $+9,58$ \\
\hline Pública & 34,86 & $+5,29$ & $+5,29$ & $+5,29$ & $+5,29$ & $+5,29$ \\
\hline
\end{tabular}

*Dado $\mathrm{x}$ como a quantidade de água consumida. 
Note que o número de pessoas atendidas pelo abastecimento de água em cada trecho de tubulação não foi considerado diretamente, por ser difícil sua mensuração em setores comerciais, hospitalares ou industriais. Contudo, sua influência é verificada, indiretamente, pelo consumo de cada estabelecimento.

\subsection{Escolha do método multicritério}

Primeiramente, para que o índice seja utilizado no modelo matemático de setorização para manobras é importante que o método de agregação multicritério utilizado seja capaz de resultar num valor numérico, de forma a ordenar as alternativas. Assim, os métodos que realizam seleção e classificação das alternativas não são adequados.

Outra questão relevante é sobre o processo de eliciação de parâmetros. Todos os métodos têm algum(s) parâmetro(s) a ser(em) eliciado(s) como, por exemplo, as constantes de escala. É importante que o método escolhido tenha um processo de eliciação estruturado para tornar mais fácil para o decisor expressar suas preferências. É importante, também, que esse procedimento seja simples, ou seja, não demande do decisor conhecimentos específicos sobre o método escolhido.

Além disso, como as redes de distribuição de água não são estáticas, ou seja, sofrem constantes alterações e/ou ampliações, os métodos que realizam uma comparação entre pares de alternativas podem não ser apropriados, pois quando adicionado ou removido um trecho de tubulação, a avaliação numérica dos demais trechos pode se alterar.

No caso do abastecimento de água, devido ao caráter social, um processo de trade-off entre o que é melhor para a empresa abastecedora e o que é melhor para os usuários é interessante. Assim, um método de agregação multicritério que realize uma compensação entre as avaliações pode ser mais apropriado.

Por fim, é de fundamental importância a verificação e a exploração de determinadas propriedades de independência que podem existir entre as preferências do decisor para vários critérios diferentes (KEENEY; RAIFFA, 1976). No caso analisado é considerado que a empresa abastecedora irá sempre preferir mais tarifação $\left(\mathrm{Cr}_{3}\right)$ independentemente da quantidade de unidades consumidoras, ou seja, a quantidade delas não altera a preferência desse decisor no critério valor da tarifa.

Portanto, a partir das reflexões acima, destaca-se o método SMARTER (Simple Multi-Attribute Rating Technique Extended to Ranking) para agregar as avaliações na geração do índice de priorização proposto, uma vez que ele atende a todos os requisitos levantados. É importante deixar claro que outros métodos de ordenação podem ser considerados, a depender das características específicas de cada rede de distribuição de água, bem como das preferências e racionalidade dos decisores envolvidos.

\subsection{Método SMARTER}

A primeira etapa do uso do SMARTER é definir os critérios e alternativas (ver item 4.1). Na sequência, é necessário que todas as avaliações estejam na mesma escala de medida. Por esse motivo é importante realizar um procedimento de normalização. De acordo com Almeida (2011), a função valor $v_{j}\left(a_{i}\right)$ fornece a avaliação de cada alternativa $i$ para cada critério $j$, em que todos os valores de $v_{j}\left(a_{i}\right)>0$. Assim, destacam-se, basicamente, três procedimentos de normalização, que são:

- Procedimento 1: Pode ser interpretado como uma percentagem do valor máximo de $v_{j}\left(a_{i}\right)$ e indica a distância da principal alternativa, conforme Equação 1.

$v_{j}^{\prime}\left(a_{i}\right)=v_{j}\left(a_{i}\right) /\left[\operatorname{Max} v_{j}\left(a_{i}\right)\right]$

- Procedimento 2: Pode ser interpretado como uma percentagem da faixa Max $v_{j}\left(a_{i}\right)-\operatorname{Min} v_{j}\left(a_{i}\right)$, conforme Equação 2. Observa-se que o zero significa apenas o mínimo.

$v_{j}^{\prime}\left(a_{i}\right)=\left[v_{j}\left(a_{i}\right)-\operatorname{Min} v_{j}\left(a_{i}\right)\right] /\left[\operatorname{Max} v_{j}\left(a_{i}\right)-\operatorname{Min} v_{j}\left(a_{i}\right)\right]$

- Procedimento 3: Pode ser interpretado como uma percentagem do total $\sum_{\mathrm{i}} \mathrm{v}_{\mathrm{j}}\left(a_{\mathrm{i}}\right)$, conforme Equação 3.

$v_{j}^{\prime}\left(a_{i}\right)=v_{j}\left(a_{i}\right) /\left[\sum_{i} v_{j}\left(a_{i}\right)\right]$

A proporcionalidade é mantida nos procedimentos 1 e 3 (escala de razão). No segundo procedimento (escala intervalar), a proporcionalidade não é mantida, ou seja, $v_{j}^{\prime}\left(a_{\mathrm{j}}\right) / v_{\mathrm{j}}^{\prime}\left(a_{\mathrm{k}}\right)$ não é necessariamente igual a $v_{\mathrm{j}}\left(a_{\mathrm{i}}\right) /$ $v_{j}\left(a_{k}\right)$. A ordenação das alternativas para cada critério será a mesma para qualquer um dos três procedimentos de normalização. No entanto, para a comparação geral entre as alternativas não (considerando todos os critérios, com avaliação intercritérios). Portanto, quando se muda o procedimento de normalização, devem-se avaliar as constantes de escala para essa nova situação. Além disso, nas equações apresentadas deseja-se maximizar o resultado; nos casos em que se deseja a minimização deve se fazer a inversão pela Equação 4.

$v_{j}^{\prime}\left(a_{i}\right)=1 / v_{j}\left(a_{i}\right)$

Para definir qual procedimento de normalização adotar é preciso entender melhor as escalas de medida 
de cada critério. Analisando os critérios levantados tem-se $\mathrm{Cr}_{1}$ (número de economias), $\mathrm{Cr}_{3}$ (tarifas da água) e $\mathrm{Cr}_{4}$ (consumo), que estão registrados em uma escala de razão, uma vez que o ponto zero é claramente uma ausência de valor. Enquanto o critério $\mathrm{Cr}_{2}$ (tipo de setor) registra as alternativas em uma escala intervalar, na qual existe uma ordem clara, com a distância entre as avaliações definida, e o ponto zero é apenas um ponto de referência.

Assim sendo, respeitando a hierarquia das medidas e tomando-se todas as avaliações na mesma escala (intervalar), o segundo procedimento de normalização será adotado. Os valores serão expressos entre 0 e 1 , em que 0 é a pior alternativa e 1 , a melhor alternativa em cada critério, independentemente se o seu objetivo é maximizar ou minimizar aquele critério. No caso deste trabalho busca-se a maximização em todos os critérios.

Posteriormente à normalização, é importante determinar a importância de cada critério. Assim, o processo chamado "swing dos pesos" consiste em incluir uma alternativa hipotética que tem o pior desempenho em todos os critérios. Então, algumas perguntas são feitas ao decisor, tal como: "Dada a oportunidade de substituir a avaliação desta alternativa para o melhor desempenho em apenas um critério, qual critério você escolheria?" (FONTANA; MORAIS; ALMEIDA, 2011, p.575).

Esse processo é continuado até que todos os critérios tenham sido avaliados e seja possível ordená-los por meio dessa avaliação das consequências resultantes do "swing dos pesos".

Após esse processo, o SMARTER utiliza valores predeterminados chamados Rank Order Centroid weights (ROC) para estabelecer as constantes de escala. Edwards e Barron (1994) afirmam que uma decisão baseada na curva ROC apresenta ganhos de $98 \%$ a 99\%, em média, na função valor obtida através do levantamento completo das constantes de escala.

Então, dado $m$ critérios, tal que $j=\{1,2,3, \ldots$, $m\}$, e uma ordem dos critérios determinada pelo DM no procedimento anterior igual a: $w_{1} \succ w_{2} \succ w_{3} \succ$ $\ldots \succ w_{\mathrm{m}}$, as constantes de escala pela curva ROC são calculadas pela equação 5 .

$$
\begin{aligned}
& W_{1}=(1+1 / 2+1 / 3+\ldots+1 / m) / m \\
& w_{2}=(1 / 2+1 / 3+\ldots+1 / m) / m \\
& w_{3}=(1 / 3+\ldots+1 / m) / m \\
& \ldots \\
& w_{\mathrm{m}}=(1 / m) / m .
\end{aligned}
$$

Por fim, o resultado pelo método SMARTER é dado através do somatório das ponderações entre as avaliações das alternativas em cada critério e a constante de escala do mesmo critério (chamada de "peso" pelos desenvolvedores do método). Assim, a função valor global para uma alternativa $A_{1}$ é expressa pela Equação 6.

$U_{A 1}=\sum_{j=1}^{m} w_{c_{r i}} * u_{A 1}\left(x_{A 1 C r i}\right)$

\section{Modelo de setorização para manobras}

Neste modelo assume-se que exista apenas um reservatório, ou seja, apenas um ponto de origem do abastecimento de água para a rede de distribuição. Além disso, para evitar o seccionamento de trechos da tubulação, uma válvula de fechamento só poderá ser localizada nos trechos de tubulação $\left(\mathrm{t}_{\mathrm{ij}}\right)$ no ponto mais próximo de um nó.

0 diâmetro dessas válvulas é o mesmo da tubulação em que a válvula é instalada. Nos diâmetros maiores, seu custo de aquisição e de instalação são bastante significativos (HELLER; PÁDUA, 2006). Assim, neste modelo, a alocação de válvulas de manobra em condutas principais não será permitida. Além disso, com uma ampliação da rede, uma conduta principal, que abastecia certo número de condutas secundárias, poderá abastecer um número maior de condutas e, assim, a válvula na conduta principal pode não atender às restrições dessa rede ampliada. Por esses e outros motivos na prática são verificadas válvulas inativas ou ineficientes.

Assim, apenas as condutas secundárias poderão ser setorizadas. Logo, tomando como base a NBR 12218/94 (ASSOCIAÇÃO..., 1994), o modelo de setorização é definido como:

$\operatorname{Min} Z=\sum_{i=1}^{n} V_{i}$

S.a.

$V_{i} \leq \sum_{j=1}^{n} t_{i j}$

$40.000 \leq\left(\sum_{i=1}^{n} \sum_{j=1}^{n} t_{i j} * a_{i j}\right) \leq 200.000$

$$
\begin{gathered}
\mathrm{e} / \mathrm{ou} \\
7.000 \leq\left(\sum_{i=1}^{n} \sum_{j=1}^{n} t_{i j} * d_{i j}\right) \leq 35.000
\end{gathered}
$$

$$
\begin{gathered}
\mathrm{e} / \mathrm{ou} \\
600 \leq\left(\sum_{i=1}^{n} \sum_{j=1}^{n} t_{i j} u_{i j}\right) \leq 3.000
\end{gathered}
$$

$\sum \sigma_{i j} \leq w$ 
$V_{i}$ e $\sigma_{i j} \geq 0$

em que: $V_{\mathrm{i}}$ - Número de válvulas no nó $i ; t_{\mathrm{ij}}-$ Trecho que liga o nó $i$ ao $j$ (informa se existe ligação entre $i$ e $j) ; a_{\mathrm{ij}}$ - Área de abastecimento compreendida pelo trecho $t_{\mathrm{ij}} ; d_{\mathrm{ij}}$ - Comprimento do trecho $t_{\mathrm{ij}} ; u_{\mathrm{ij}}$ - Número de unidades consumidoras (economias) que estão entre o nó $i$ e o $j$; $\sigma_{\mathrm{ij}}$ - Índice de priorização do trecho $t_{\mathrm{ij}}$; $w$ - Limite superior ao somatório dos $\sigma_{\mathrm{ij}}$.

A função objetivo (7) pretender minimizar o número de válvulas de manobra nos nós $i$, tal que $i=\{1,2, \ldots, n\}$. A restrição (8) estabelece que o número de válvulas no nó $i$ deve ser menor ou igual ao número de tubulações que se interligam naquele nó. A restrição (9) estabelece um limite para a área total de um setor de manobra. A restrição (10) estabelece um limite para a extensão total de um setor de manobra. A restrição (11) estabelece um limite para o número total de unidades consumidoras em um setor de manobra. As restrições (9), (10) e (11) podem ser todas ou apenas uma atendidas, elas são formuladas a partir da NBR 12218/94 (ASSOCIAÇÃO..., 1994). A restrição (12) estabelece um limite superior ao somatório dos índices de priorização dos trechos constituintes de um setor de manobras. Por fim, a restrição (13) estabelece a não negatividade do número de válvulas e do índice de priorização.

$\mathrm{Na}$ prática, em redes ramificadas e mistas é comum a presença de setores menores dentro de setores maiores, ou seja, é possível fechar uma área menor dentro de uma área maior isolada por válvulas. Considere, na Figura 1, a alocação de uma válvula $\left(\mathrm{V}_{1}\right)$ após o nó 2: um setor será gerado. Se, em seguida, uma válvula $\left(\mathrm{V}_{2}\right)$ for alocada após o nó 5, no trecho [5], um setor menor será isolado. Ele é considerado setor menor, porque sempre que a válvula $\mathrm{V}_{1}$ for acionada, os trechos após $\mathrm{V}_{2}$ também serão desabastecidos.

Conforme normas da NBR $12218 / 94$ (ASSOCIAÇÃO..., 1994), nas condutas secundárias, uma válvula junto ao ponto de ligação a condutas principais deverá ser prevista. $0 \mathrm{u}$ seja, se $t_{\mathrm{ij}}$ for tronco e estiver interligado no nó $j$ a condutas secundárias, deve-se prever uma válvula de manobra após o nó $i$. Porém Heller e Pádua (2006) argumentam que essa condição pode demasiar a utilização de válvulas. Assim, o modelo proposto será simulado com o atendimento dessa norma, bem como simulará a busca pela solução sem o atendimento desta norma, a priori.

\subsection{Algoritmo do modelo de setorização para manobras}

Com base nas informações apresentadas, o algoritmo de resolução do problema é sumarizado pela Figura 2.

Passo 1: Para iniciar o procedimento deve-se considerar uma solução inicial, ou seja, uma solução com determinado número de válvulas e sua localização estabelecidos a priori. Existem três possibilidades para isto:

- Admitir a rede como um setor de manobra, uma vez que o reservatório é capaz de fechar o fluxo de água;

- Admitir as normas da NBR 12218/1994 (ASSOCIAÇÃO..., 1994), alocando as válvulas à rede e tornando-as fixas no modelo;

- Em redes existentes, considerar as válvulas que possam existir na rede e também fixá-las no modelo.

Passo 2: Verifique se o fluxo de água

- Para um número de válvulas = 0 (ir para o passo 3);

- Para um número de válvulas > 0;

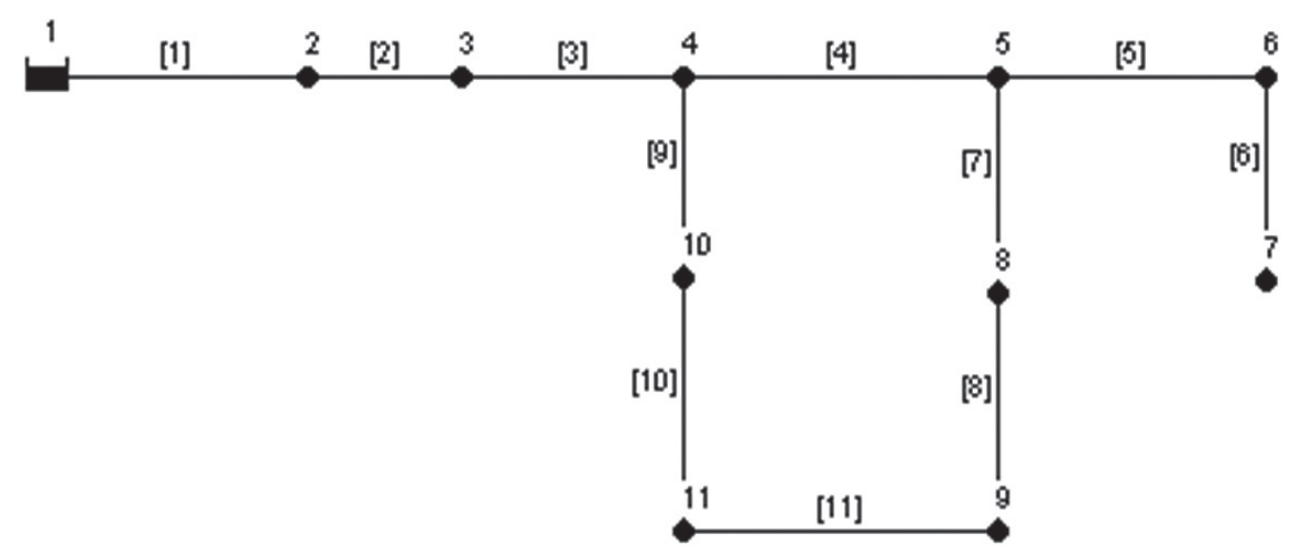

Figura 1. llustração para setorização. 


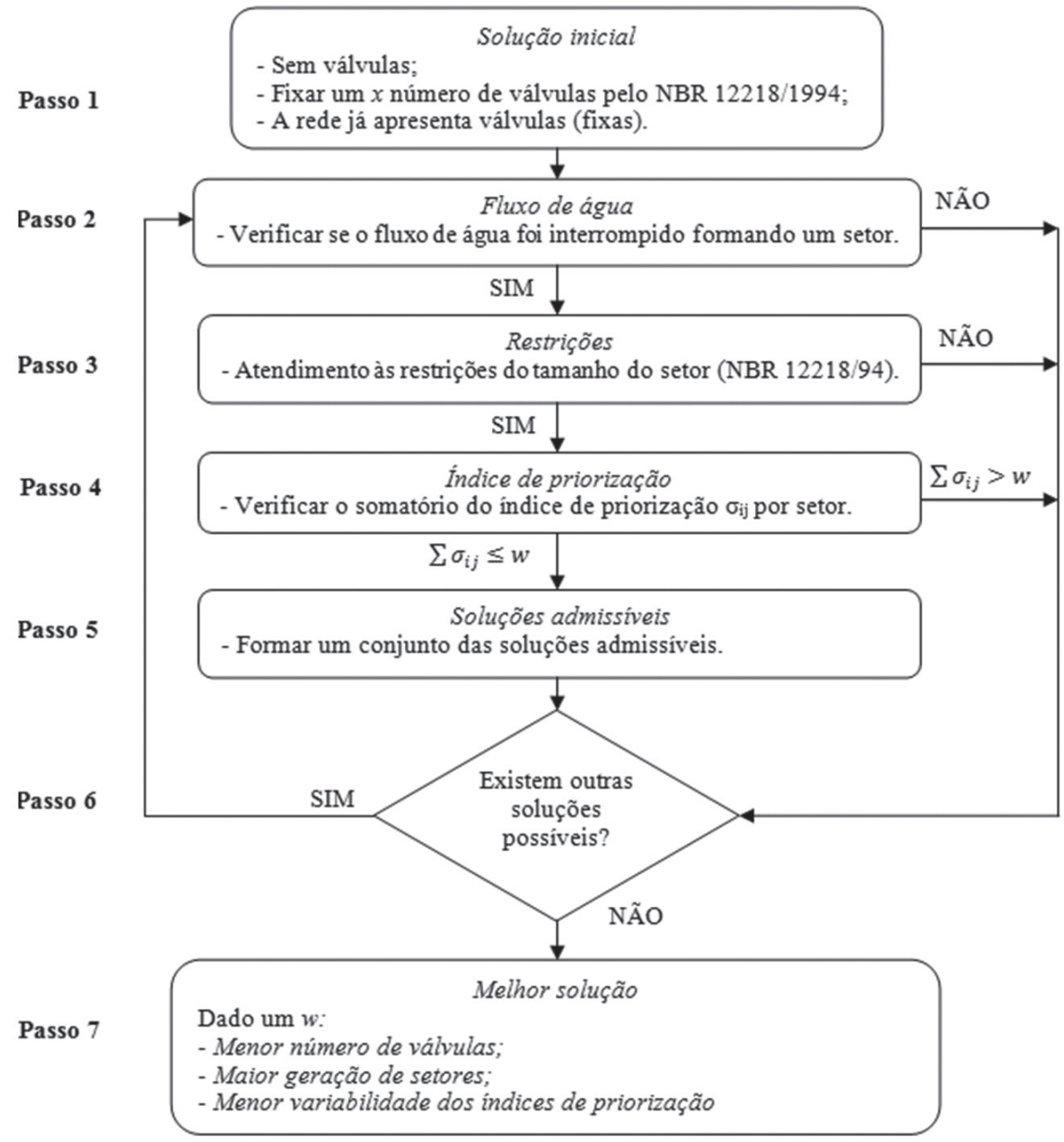

Figura 2. Sumarização do modelo proposto.

- Verifique o fluxo da água após uma válvula ou entre válvulas;

- Se o fluxo de água é isolado = solução admissível (ir para o passo 3);

- Caso contrário = solução não admissível (ir para o passo 6).

Passo 3: Dadas as restrições do modelo quando à área (a), comprimento (b) e número de economias (c), verifique se pelo menos uma delas é atendida pelo setor formado. Caso (b) e (c) estejam abaixo do seu limite inferior, desconsidere o limite superior para a área (a).

- Se sim = setor admissível (passe para o passo 4);

- Caso contrário = Setor não admissível (ir para o passo 6).

Passo 4: Verificar o somatório do índice de priorização $\sigma_{\mathrm{ij}}$ para cada setor formado. Note que uma vez que cada trecho pode constituir um setor, o valor de $w$ não pode ser menor que o maior dos indices de priorização dos trechos $\left(\sigma_{\mathrm{ij}}\right)$.

- Se $\sum \sigma_{i j} \leq w$ em todos os setores, e também nos trechos não setorizados = solução admissível (ir para o passo 5).

- Se existirem setores menores, considerar: $\sum \sigma_{\text {setor_maior }}=\sum \sigma_{\text {total_setor }}-\sum \sigma_{\text {setor_menor }}$;

- Caso contrário $\left(\sum \sigma_{i j}>w\right)=$ solução não admissível (ir para o passo 6).

Passo 5: Formar um conjunto com todas as soluções admissiveis encontradas.

Passo 6: Verificar a existência de soluções admissíveis:

- Sobre a solução inicial, alocar uma ou mais válvulas na rede, alternado sua localização em cada rodada do modelo (volte ao passo 2). 
- Após todas as possíveis soluções serem analisadas, ir para o passo 7.

Passo 7: A solução do problema será aquela solução admissível que resultar na menor utilização de válvulas de fechamento. Em caso de haver mais de uma solução com o menor número de válvulas:

- Preferir aquela solução que resultou no maior número de setores;

- Persistindo o empate, escolher aquela solução em que a variância da média dos $\sum \sigma_{i j}$ dos setores seja a menor, indicando uma melhor distribuição dos índices de priorização pelos setores formados. Caso haja algum setor menor dentro de outro setor, haverá um $\sum \sigma_{\text {setor_menor }}$ (considerado como setor) e um $\sum \sigma_{\text {setor }}=\sum \sigma_{\text {setor_total }-} \sum \sigma_{\text {setor_menor }}$. Assim, seja o número de setores $i=\{1,2, \ldots, n\}$ e o número de trechos de um setor $j=\{1,2, \ldots, m\}$, tem-se:

$$
M_{i}=\frac{\sum_{j=1}^{m} \sigma_{\text {setor } i}}{\sum_{j=1}^{m} t_{\text {setor } i}}
$$

$\bar{M}=\frac{1}{n} \sum_{i=1}^{n} M_{i}$

$\operatorname{Var}=\frac{1}{n} \sum_{i=1}^{n}\left(M_{i}-\bar{M}\right)^{2}$

Desta forma, a equação (14) calcula o índice de priorização médio por trecho de tubulação de um setor; a equação (15) calcula a média dos índices de priorização médios; por fim, a equação (16) calcula a variância da média dos índices de priorização médios. Note que em uma solução inicial sem válvulas fixas, os trechos não setorizados serão adicionados aos trechos de tubulações primárias. Assim, o número de trechos primários não deve entrar na média do somatório dos índices de priorização $\left(\sum \sigma\right)$ daqueles trechos não setorizados.

- Por fim, as soluções empatadas serão consideradas tecnicamente indiferentes.

É importante destacar que o modelo deve ser usado para auxiliar na decisão, ficando a cargo da experiência dos técnicos responsáveis avaliar a efetivação da solução dada pelo modelo.

\subsection{Cálculo da área dos trechos}

Para o cálculo da área, primeiramente, pensou-se em somar a área das economias abastecidas diretamente por cada trecho, o que constituiria sua área. Porém, a área do setor de manobras não compreende apenas a área das economias, pois existem ruas, calçadas e lotes baldios que não constituem uma economia. Além disso, tubulações principais não abastecem diretamente as economias, mas fazem parte da área de abrangência de um setor de manobra.

Dessa maneira, para estimar um valor aproximado da área que represente um trecho, primeiramente deve-se delimitar a área total da rede analisada. A Figura 3 apresenta a delimitação da área para uma rede malhada.

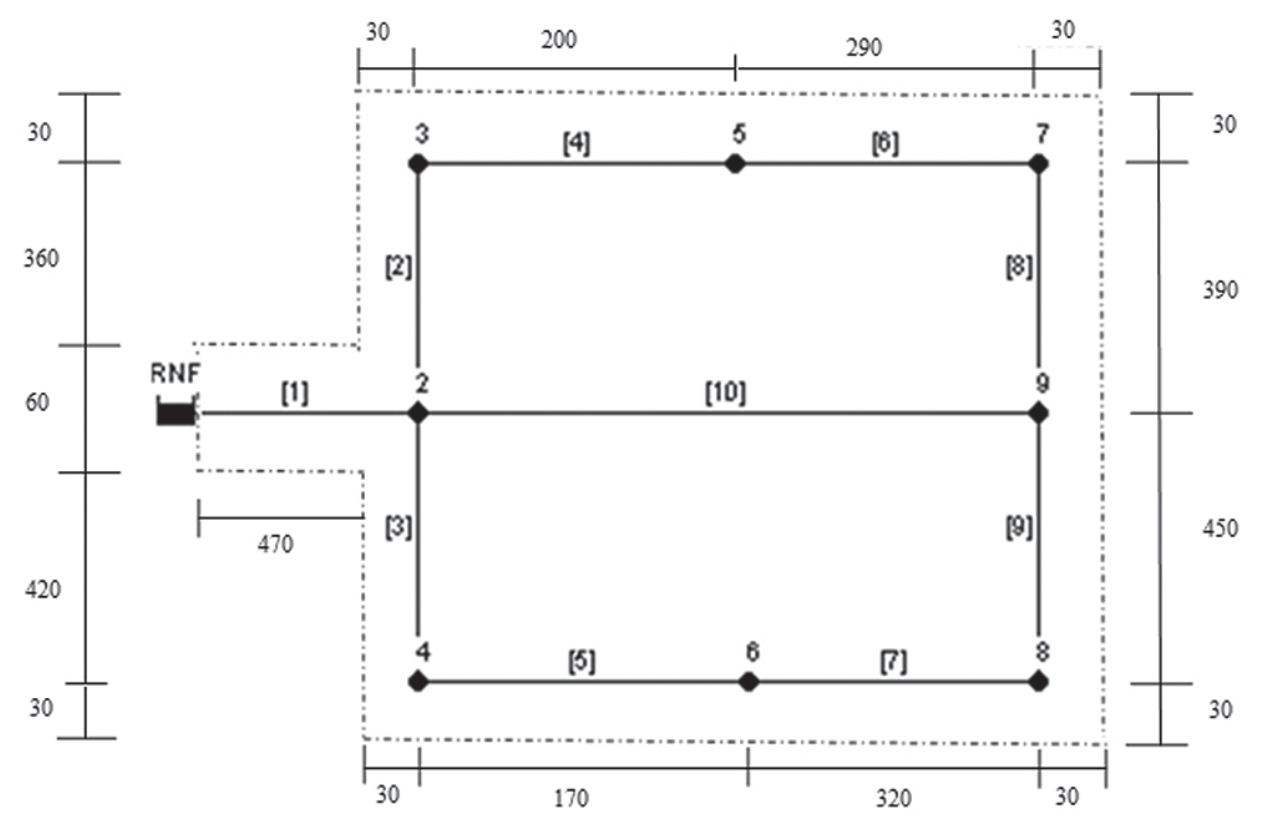

Figura 3. Cálculo da área. 
Com essa configuração, a área total da rede será: $A=470 * 60+550 * 900=523.000 \mathrm{~m}^{2}$. Assim, para estipular uma área por trecho, neste trabalho faz-se uma relação de proporcionalidade entre a área total da rede e o tamanho de cada trecho, como mostra a Tabela 3, em que o comprimento é dado em metros e a área em metros quadrados $\left(m^{2}\right)$.

É importante destacar que a área calculada retrata apenas uma representatividade, aproximada, da área de cada trecho e não a área exata.

\subsection{Exemplificação do uso do algoritmo do modelo de setorização para manobras}

Para exemplificar a aplicabilidade do algoritmo do modelo proposto será usada a rede malhada apresentada na Figura 3. Essa rede é um pequeno distrito de medida. A Figura 4 apresenta uma ilustração do comportamento do fluxo de água na rede.

$\mathrm{Na}$ Figura 3 pode se ser ver que o fluxo normal ocorre no sentido das setas preenchidas (+1). Contudo, quando ocorre algum tipo de interrupção na rede de distribuição de água, por se tratar de uma rede malhada, o sentido do fluxo pode alterar-se, conforme setas pontilhada $(-1)$. Além disso, para um setor de manobras ser fechado ou isolado, seu fluxo deve ser nulo. Note-se ainda que não existe reversão do fluxo no trecho [1], isso porque ele alimenta a rede de distribuição e, portanto, se o fluxo nele for interrompido não haverá fluxo contrário, com exceção de redes com múltiplos reservatórios, em que há chances disso acontecer. Em redes de distribuição de água ramificadas, esse fluxo contrário também não ocorre.

Os dados necessários para aplicar o modelo são encontrados na Tabela 4. Esses dados são ilustrativos, ou seja, foram gerados a partir de uma base de dados de uma empresa brasileira distribuidora de água, bem como calculados pelos métodos aqui apresentados. Para o cálculo do índice de priorização $\left(\sigma_{\mathrm{ij}}\right)$, suponha que os responsáveis pela rede de distribuição, após

Tabela 3. Cálculo da área.

\begin{tabular}{cccc}
\hline Trecho & $\begin{array}{c}\text { Comprimento }-(\mathrm{m}) \\
\text { (a) }\end{array}$ & $\begin{array}{c}\%(\mathrm{c})=(\mathrm{a}) / \\
(\mathrm{b})\end{array}$ & $\begin{array}{c}\text { Área }-\left(\mathrm{m}^{2}\right) \\
\mathrm{A}_{\mathrm{ij}}=\mathrm{A}^{*}(\mathrm{c})\end{array}$ \\
\hline$[1]$ & 500 & 0,136986 & $71.643,84$ \\
{$[2]$} & 390 & 0,106849 & $55.882,19$ \\
{$[3]$} & 450 & 0,123288 & $64.479,45$ \\
{$[4]$} & 200 & 0,054795 & $28.657,53$ \\
{$[5]$} & 170 & 0,046575 & $24.358,90$ \\
{$[6]$} & 290 & 0,079452 & $41.553,42$ \\
{$[7]$} & 320 & 0,087671 & $45.852,05$ \\
{$[8]$} & 390 & 0,106849 & $55.882,19$ \\
{$[9]$} & 450 & 0,123288 & $64.479,45$ \\
{$[10]$} & 490 & 0,134247 & $70.210,96$ \\
& (b) $\sum=3650 m$ & 1,000000 & $A=523.000,00$ \\
& & & $m^{2}$ \\
\hline
\end{tabular}

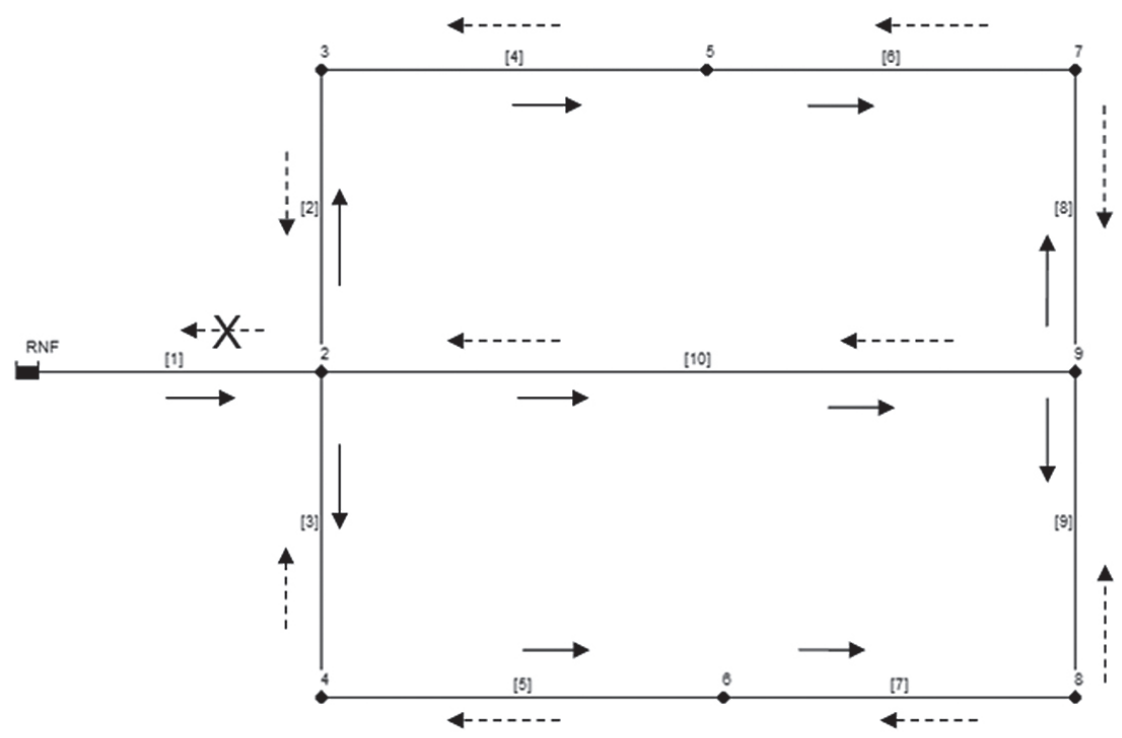

$$
\begin{aligned}
& \text { Legenda: } \\
& \longrightarrow \text { Fluxo em sentido normal (sem interrupções) }=+1 \\
& \text { intemupção (ex. válvula de fechamento acionada) }=-1
\end{aligned}
$$

Figura 4. Fluxo da rede malhada. 
Tabela 4. Exemplificação do modelo de setorização para manobra em rede malhada.

\begin{tabular}{|c|c|c|c|c|c|c|c|c|c|}
\hline *Trecho & $\begin{array}{l}\text { Nó } \\
\text { (i) }\end{array}$ & $\begin{array}{l}\text { Nó } \\
\text { (j) }\end{array}$ & Setor (un.) & $\begin{array}{c}\text { Economias } \\
u_{\mathrm{ij}}\end{array}$ & Tarifa (R\$) & $\begin{array}{c}\text { Consumo } \\
\left(\mathrm{m}^{3}\right)\end{array}$ & $\begin{array}{c}\text { Índice } \\
\sigma_{i j} \\
\end{array}$ & $\begin{array}{c}\text { Área }(\mathrm{m} 2) \\
a_{\mathrm{ij}} \\
\end{array}$ & $\begin{array}{c}\text { Comp. (m) } \\
\mathrm{d}_{\mathrm{ij}}\end{array}$ \\
\hline [1] & RNF & 2 & - & - & - & - & 0,000 & $71.643,84$ & 500 \\
\hline$[2]$ & 2 & 3 & 4 & 11 & $8.970,00$ & 1.430 & 0,153 & $55.882,19$ & 390 \\
\hline [3] & 2 & 4 & 4 & 12 & $10.210,00$ & 1.600 & 0,188 & $64.479,45$ & 450 \\
\hline [4] & 3 & 5 & 5 & 6 & $3.855,00$ & 700 & 0,260 & $28.657,53$ & 200 \\
\hline$[5]$ & 4 & 6 & 4 & 15 & $9.090,00$ & 1.650 & 0,189 & $24.358,90$ & 170 \\
\hline$[6]$ & 5 & 7 & 4 & 17 & $15.860,00$ & 2.400 & 0,354 & $41.553,42$ & 290 \\
\hline [7] & 6 & 8 & 6 & 18 & $22.240,00$ & 2.450 & 0,977 & $45.852,05$ & 320 \\
\hline [8] & 9 & 7 & 5 & 20 & $18.170,00$ & 2.640 & 0,680 & $55.882,19$ & 390 \\
\hline [9] & 9 & 8 & 4 & 11 & $8.135,00$ & 1.350 & 0,134 & $64.479,45$ & 450 \\
\hline [10] & 2 & 9 & 5 & 12 & $16.500,00$ & 1.850 & 0,560 & $70.210,96$ & 490 \\
\hline
\end{tabular}

*Rede malhada com apenas o trecho [1] é um tronco.

o procedimento de "swing dos pesos", ordenaram os critérios da seguinte maneira: $\mathrm{Cr} 2$ - Tipo de setor $\succ \mathrm{Cr} 4-$ Consumo $\succ \mathrm{Cr} 3-$ Valor da tarifa $\succ$ $\mathrm{Cr} 1$ - Número de economias. Assim, usando-se a curva ROC, conforme a equação (5), a constante de escala para cada critério será: $w_{1}=0,5208 ; w_{2}=0,2708$; $W_{3}=0,1458 ;$ e $w_{4}=0,0650$.

Considere o atendimento prévio da NBR 12218/1994 (ASSOCIAÇÃO..., 1994) para a solução inicial. Dessa forma tem-se a formação do setor $\left(\mathrm{S}_{0}\right)$ com uma válvula $\mathrm{V}_{1}$ após o nó 2 no trecho [2], uma válvula $V_{2}$ após o nó 2 no trecho [3] e outra $V_{3}$ após o nó 2 no trecho [3]. Assim, tem-se setor $\mathrm{S}_{0}=\sum \sigma_{\text {setor } 0}=3,495$ e $\Sigma \sigma=0,000$ para os trechos remanescentes ([1]). Portanto, para um $w \geq 3,495$, esse setor será aquele com a menor utilização de válvulas e, portanto, a melhor solução.

Considerando fixas as válvulas anteriores, busca-se uma solução para um $w<3,495$. Seguindo o algoritmo devem-se setorizar as tubulações adicionando uma válvula, duas, três e assim por diante, verificando-se todas as possíveis soluções.

Com base nas soluções deve-se checar o fluxo de água da seguinte maneira: suponha, como exemplo, a adição de duas válvulas no trecho [9], uma localizada no nó $8\left(\mathrm{~V}_{4}\right)$ e outra no nó $9\left(\mathrm{~V}_{5}\right)$. Em seguida, deixe essas válvulas permanentemente fechadas. Análise se os trechos que não estão nesse setor estão sendo abastecidos. Nesse exemplo, eles estão e, portanto, a solução é admissivel. Em seguida, permanecendo fechadas as válvulas $V_{4}$ e $V_{5}$, teste o fechamento das demais válvulas já colocadas na rede (solução inicial ou atual). Note-se que ao se fecharem as válvulas $\mathrm{V}_{1}$ e $\mathrm{V}_{3}$ o fluxo de água para os trechos $[2]+[4]+[6]+[8]+[10]$ é interrompido, enquanto o fluxo para os trechos [3], [5] e [7] é interrompido ao fechar-se $\mathrm{V}_{2}$. Portanto, podem-se considerar três setores para essa rede. Eles não caracterizam setores menores, pois o isolamento de cada um, individualmente, não afeta os demais, ou seja, são independentes.
Assim, deve-se verificar se todos os setores gerados atendem as normas quanto ao comprimento ou área ou número de economias. No exemplo, todos os setores atendem a área mínima. Em seguida, é feita a soma dos índices de priorização dos setores. Nesse caso tem-se: $\operatorname{setor}\left(\mathrm{S}_{1}\right)=\sum \sigma_{\text {setor } 1}=[9]=0,134$; setor $\left(\mathrm{S}_{2}\right)=\sum \sigma_{\text {setor } 2}=[3]+[5]+[7]=1,354$; e setor $\left(\mathrm{S}_{3}\right)=\sum \sigma_{\text {setor } 3}=[2]+[4]+[6]+[8]+[10]=2,007$. Portanto, para $2,007 \leq w<3,495$, a solução é admissivel. Procede-se dessa maneira para todas as soluções possíveis. Assim, considerando as 3 válvulas iniciais, a Tabela 5 resume as soluções possíveis adicionando-se apenas 1 válvula. Note-se que nenhum setor menor foi gerado nas soluções admissíveis. Esse ponto é uma vantagem das redes malhadas em relação às redes ramificadas/mistas.

Contudo, deve-se determinar qual solução é a melhor dentre as possíveis soluções. De acordo com o modelo, a melhor solução é aquela que atende ao limite $w$ com o menor número de válvulas, seguida daquela que apresentar o maior número de setores gerados com o mesmo número de válvulas e, por fim, a melhor solução é aquela que apresentar a menor variância entre a distribuição dos $\sigma$ entre os setores. A Tabela 6 resume as melhores soluções para esse exemplo por intervalo do parâmetro $w$.

Ao considerar que quanto maior o grau de priorização de um trecho maior será o impacto gerado quando uma interrupção do abastecimento de água ocorrer, uma distribuição desses trechos entre os setores de manobra pode minimizar esse impacto. Assim, as soluções que foram consideradas como as melhores são, justamente, aquelas que conseguiram distribuir melhor esse impacto por setor, de acordo com seu índice de priorização.

\section{Discussões}

Na geração do índice de priorização verificou-se que um trecho com índice próximo de 1 significa 
Tabela 5. Resumo das soluções possíveis adicionando-se 1 válvula - rede malhada.

\begin{tabular}{|c|c|c|c|c|c|}
\hline \multicolumn{6}{|c|}{$\mathrm{N}^{\circ}$ de válvulas adicionais $=1$} \\
\hline Solução & Endereço & Trechos compreendidos & Solução admissivel? & $\sum \sigma_{\text {setor } 1}$ & $\sum \sigma_{\text {setor } 2}$ \\
\hline A & Nó 3/trecho [2] & [2] & Sim & 0,153 & 3,342 \\
\hline B & Nó 5/trecho [4] & {$[2]+[4]$} & Sim & 0,413 & 3,082 \\
\hline c & Nó 7/trecho [6] & {$[2]+[4]+[6]$} & Sim & 0,767 & 2,728 \\
\hline $\mathrm{D}$ & Nó 9/trecho [8] & {$[2]+[4]+[6]+[8]$} & Sim & 1,447 & 2,048 \\
\hline E & Nó 4/trecho [3] & [3] & Sim & 0,188 & 3,307 \\
\hline $\mathrm{F}$ & Nó 6/trecho [5] & {$[3]+[5]$} & Sim & 0,377 & 3,118 \\
\hline G & Nó 8/trecho [7] & {$[3]+[5]+[7]$} & Sim & 1,354 & 2,141 \\
\hline $\mathrm{H}$ & Nó 9/trecho [9] & {$[3]+[5]+[7]+[9]$} & Sim & 1,488 & 2,007 \\
\hline 1 & Nó 9/trecho [10] & [10] & Sim & 0,560 & 2,935 \\
\hline Solução & $\mathrm{N}^{\circ}$ válvulas/setor & $\mathrm{N}^{\circ}$ de trechos $\left(\mathrm{t}_{\mathrm{ij}}\right) /$ setor & $" M_{1}$ & $" \mathrm{M}_{2}$ & Var \\
\hline A & $4 / 2$ & $1 / 8$ & 0,15300 & 0,417750 & 0,017523 \\
\hline B & $4 / 2$ & $2 / 7$ & 0,20650 & 0,440286 & 0,013664 \\
\hline C & $4 / 2$ & $3 / 6$ & 0,25567 & 0,454667 & 0,009900 \\
\hline D & $4 / 2$ & $4 / 5$ & 0,36175 & 0,409600 & 0,000572 \\
\hline E & $4 / 2$ & $1 / 8$ & 0,18800 & 0,413375 & 0,012698 \\
\hline $\mathrm{F}$ & $4 / 2$ & $2 / 7$ & 0,18850 & 0,445429 & 0,016503 \\
\hline G & $4 / 2$ & $3 / 6$ & 0,45133 & 0,356833 & 0,002233 \\
\hline $\mathrm{H}$ & $4 / 2$ & $4 / 5$ & 0,37200 & 0,401400 & 0,000216 \\
\hline 1 & $4 / 2$ & $1 / 8$ & 0,56000 & 0,366875 & 0,009324 \\
\hline
\end{tabular}

*Em que: M1 é a média do setor 1; M2 a média do setor 2; e Var é a variância entre M1 e M2.

Tabela 6. Resumo das melhores soluções por intervalo do parâmetro $w$ - rede malhada.

\begin{tabular}{cccc}
\hline Para: $w$ & Solução & $\mathrm{N}^{\circ}$ válvulas & $\mathrm{N}^{\circ}$ setores \\
\hline$\geq 3,495$ & {$[2] / 2,[3] / 2$ e $[10] / 2$} & 3 & 1 \\
$2,007 \leq w<3,495$ & {$[2] / 2,[3] / 2,[9] / 9$ e $[10] / 2$} & 4 & 2 \\
$1,447 \leq w<2,007$ & {$[2] / 2,[3] / 2,9 /[8], 8 /[9] \mathrm{e}[10] / 2$} & 5 & 3 \\
$1,374 \leq w \leq 1,447$ & {$[2] / 2,[3] / 2,8 /[9], 7 /[8] \mathrm{e}[10] / 2$} & 5 & 3 \\
\hline
\end{tabular}

que um desabastecimento de água naquele trecho tem um impacto maior do que em um trecho com índice próximo de 0 , seja pelos setores econômicos localizados nesse trecho, seja pelo elevado consumo de água. Desse modo, é necessário realizar uma análise de sensibilidade sobre alguns parâmetros do modelo, tal como as constantes de escala do SMARTER, a fim de verificar possíveis alterações no índice de priorização, o qual é um dado de entrada para o modelo de setorização.

No modelo de setorização, o valor do parâmetro $w$ estabelece uma relação de nível de serviço oferecido por setor. A formação de setores baseados nesse parâmetro consegue limitar o número de unidades consumidoras com elevado impacto presentes em um setor. Dessa maneira, quando uma interrupção de água for inevitável, o impacto total pode ser menor do que em uma setorização que não leve em consideração as características das unidades consumidoras.

Problemas de alocação de válvulas, seja de fechamento ou reguladoras, em que é preciso verificar todas as possíveis combinações de número de válvulas, como é o caso do modelo proposto, exigem métodos de resolução que minimizem o tempo computacional. Por esse motivo, Ozger e Mays (2004) afirmam que o uso de técnicas de otimização heurísticas é eficiente para encontrar uma solução "quase ótima" em problemas que envolvem redes de distribuição de água.

Contudo, é importante salientar que o modelo proposto permite aos decisores a tomada de decisão sobre a localização das válvulas baseada no impacto da falta de água nos trechos da rede com a alocação mínima de válvulas possível e não mais de maneira intuitiva, ou por regras gerais pré-estabelecidas que não considerem as características específicas de cada rede de distribuição.

\section{Considerações finais}

A setorização da rede torna mais fácil qualquer atividade de manutenção, além de atingir um número menor de unidades de consumo. Quanto menor o setor de manobras, menores serão as chances da ocorrência de interrupções no abastecimento. Porém 
a demasiada utilização de válvulas de fechamento pode encarecer o sistema, seja por sua implantação, seja por sua manutenção.

Para que seja possível aplicar este modelo de setorização, um índice de priorização dos trechos da tubulação da rede de distribuição foi proposto. Esse índice é uma medida para avaliar o impacto da falta de água nas unidades consumidoras. Na geração do índice verificaram-se algumas características importantes do problema, que levaram à escolha do método SMARTER. Esse método, além de atender aos requisitos do problema, ainda se destaca pelas constantes de escala dadas pela curva ROC, o que facilita o processo de eliciação das preferências do(s) decisor(es).

No conjunto de soluções admissíveis sempre existirá uma solução que é mais adequada a cada intervalo do parâmetro $w$. Contudo, estabelecer um valor para esse parâmetro pode ser uma tarefa complicada para os responsáveis pela rede de distribuição de água. Uma alternativa à resolução desse problema é realizar uma análise custo versus benefício com as melhores soluções resultantes da variação do parâmetro $w$. Essa análise ainda está em estudo.

Além disso, o isolamento de parte da tubulação pode alterar a pressão e vazão da água em determinados trechos durante uma manutenção. Este trabalho concentrou-se apenas na alocação das válvulas de isolamento e sua localização não depende da alteração de pressão. Assim, após a definição dessas válvulas, um estudo de possível alteração na pressão e vazão deve ser feito, bem como estabelecida a necessidade de adição de válvulas reguladoras de pressão. Dessa maneira, o modelo pode ser agregado a uma plataforma de designer de redes de distribuição de água.

\section{Referências}

ASSOCIAÇÃO BRASILEIRA DE NORMAS TÉCNICAS - ABNT. NBR 12218/1994: Projeto de rede de distribuição de água para abastecimento público. ABNT, 1994.

ALMEIDA, A. T. O conhecimento e o uso de métodos multicritério de apoio a decisão. Recife: Ed. Universitária da UFPE, 2011.

ALMEIDA, A. T.; COSTA, A. P. C. S. Aplicações com Métodos Multicritério de Apoio a Decisão. Recife: Ed. UFPE, 2003. v. 14.

BANA E COSTA, C. A.; VANSNICK, J.-C. MACBETH An Interactive Path Towards the Construction of Cardinal Value Functions. International Transactions in Operational Research, v. 1, n. 4, p. 489-500, 1994. http://dx.doi.org/10.1111/j.1475-3995.1994.00325.x

BLACK, D. The Theory of Committees and Elections. Cambridge: Cambridge University Press, 1958.

BELTON, V.; STEWART, T. J. Multiple Criteria Decision Analysis. Kluwer Academic Publishers, 2002. http:// dx.doi.org/10.1007/978-1-4615-1495-4
CHRISTODOULOU, S.; DELIGIANNI, A. A neurofuzzy decision framework for the management of water distribution networks. Water Resource Management, v. 24, p. 139156, 2010. http://dx.doi.org/10.1007/s11269-009-94412

DELGADO-GALVÁN, X. et al. An analytic hierarchy process for assessing externalities in water leakage management. Mathematical and Computer Modelling, v. 52, p. 1194-1202, 2010. http://dx.doi.org/10.1016/j. mcm.2010.03.014

EDWARDS, W.; BARRON, F. H. SMARTS and SMARTER: improved simple methods for multiattribute utility measurement. Organizational Behavior and Human Decision Processes, v. 60, p. 306-325, 1994. http:// dx.doi.org/10.1006/obhd.1994.1087

FIGUEIRA, J.; MOUSSEAU, V.; ROY, B. Chapter 4. Electre Methods. International Series in Operations Research \& Management Science, v. 78, 2006.

FONTANA, M. E.; MORAIS, D. C.; ALMEIDA, A. T. A MCDM model for urban water conservation strategies. Evolutionary Multi-Criterion Optimization Lecture Notes in Computer Science, v. 6576, p. 564-578, 2011. Disponivel em: <http://dblp.uni-trier.de/db/conf/emo/ emo2011.html\#FontanaMA11>.

GIUSTOLISI, 0.; SAVIC, D. Identification of segments and optimal isolation valve system design in water distribution networks. Urban Water Journal, v. 7, n. 1, p. 1-15, 2010. http://dx.doi.org/10.1061/41024(340)31

HELlER, L.; PÁDUA, V. L. Abastecimento de água para consumo humano. Belo Horizonte: Editora UFMG, 2006.

JUN, H. Strategic valve locations in a water distribution system. 2005. Thesis (Doctorate)-Faculty of the Virginia Polytechnic Institute and State University, 2005.

JUN, H. et al. Valve distribution and impact analysis in water distribution systems. Journal of Environmental Engineering, v. 133, n. 8, p. 790-799, 2007. http:// dx.doi.org/10.1061/(ASCE)0733-9372(2007)133:8(790)

KLAMLER, C. On the closeness aspect of three voting rules: Borda - Copeland - Maximin. Group Decision and Negotiation, v. 14, p. 233-240, 2005. http://dx.doi. org/10.1007/s10726-005-0958-3

KEENEY, R. L.; RAIFFA, H. Decision with multiple objectives: preferences and value trade-offs. John Wiley \& Sons, 1976.

KIM, J. H.; MAYS, L. W. Optimal rehabilitation model for water-distribution systems. Journal of Water Resources Planning and Management, v. 120, p. 674692, 1994. http://dx.doi.org/10.1061/(ASCE)07339496(1994)120:5(674)

LAMBERT, V.; HIRNER W. Losses from water supply systems: standard terminology and recommended performance measures. Voda l Sanitarna Tehnika, v. 32, p. 2938, 2002.

MAYS, L. W. (Org.). et al. Water distribution systems handbook. McGraw-Hill, 2000.

MORAIS, D. C.; ALMEIDA, A. T. Modelo de decisão em grupo para gerenciar perdas de água. Pesquisa Operacional, v. 26 , n. 3, p. 567-584, 2006. http://dx.doi.org/10.1590/ S0101-74382006000300007

MORAIS, D. C.; ALMEIDA, A. T. Group decision-making for leakage management strategy of water network. Resources, Conservation and Recycling, v. 52, 
p. 441-459, 2007. http://dx.doi.org/10.1016/j. resconrec.2007.06.008

MORAIS, D. C.; CAVALCANTE, C. A. V.; ALMEIDA, A. T. Prioritization of areas of loss control in water distribution networks. Pesquisa Operacional, v. 30, n. 1, p. 1532, 2010.

MOUNCE, S. R.; BOXALL, J. B.; MACHELL, J. Development and verification of an online artificial intelligence system for detection of bursts and other abnormal flows. Journal of Water Resources Planning and Management-Asce, v. 136, p. 309-318, 2010. http://dx.doi.org/10.1061/ (ASCE)WR. 1943-5452.0000030

NICOLINI, M.; ZOVATTO, L. Optimal Location and Control of Pressure Reducing Valves in Water Networks. Journal of Water Resources Planning and Management-Asce, v. 135, n. 3, p. 178-187, 2009. http://dx.doi.org/10.1061/ (ASCE)0733-9496(2009)135:3(178)

NURMI, H. Voting Procedures: A summary analysis. British Journal of Operational Research, v. 82, p. 522-539, 1983. http://dx.doi.org/10.1017/S0007123400003215

OZGER, S.; MAYS, L. W. Chapter 7 - Optimal location of isolation valves in water distribution systems: a reliability/ optimization approach. In: MAYS, L. W. Water resource systems management tools. McGraw-Hill Professional Engineering, 2004.

RAMOS, H. et al. Leakage control policy within operating management tools. Computer and Control for Water Industry, p. 61-73, 2001.

ROY, B. Multicriteria methodology for decision aiding. Netherlands: Kluwer Academic Publishers, 1996. http:// dx.doi.org/10.1007/978-1-4757-2500-1

ROY, B.; BERTIER, P. La méthode Electre 11 - une application au média-planning. Operational Research, p. 291-302, 1973. http://dx.doi.org/10.1590/S010365132010005000012

SAATY, T. L. The Analytic Hierarchy Process. New York: McGraw-Hill, 1980.

TROJAN, F.; MORAIS, D. C. Using Electre TRI to support maintenance of water distribution networks. Pesquisa Operacional, v. 32, n. 2, p. 423-442, 2012a. http:// dx.doi.org/10.1590/S0101-74382012005000013

TROJAN, F.; MORAIS, D. C. Prioritizing alternatives for maintenance of water distribution networks: a group decision approach. Water S.A., v. 38, p. 555-514, 2012 b. http://dx.doi.org/10.4314/wsa.v38i4.11

VINCKE, P. Multicriteria decision aid. Bruxelles: Jonh Wiley \& Sons, 1992.

WU, Z. Y.; SAGE, P.; TURTLE, D. Pressure-dependent leak detection model and its application to a district water system. Journal of Water Resources Planning and Management-Asce, v. 136, p. 116-128, 2010. http:// dx.doi.org/10.1061/(ASCE)0733-9496(2010)136:1(116)

YOUNG, H. P. Condorcet's Theory of Voting. The American Political Science Review, v. 82, n. 4, p. 1231-1244, 1988. http://dx.doi.org/10.2307/1961757

YSUSI, M. A. System Design: An Overview. In: MAYS, L.W. Water Distribution System Design. New York: McGrawHill, 2000.

\section{Agradecimentos}

Este trabalho foi desenvolvido com suporte parcial da Capes e do CNPq. Os autores agradecem também as valiosas sugestões feitas pelos revisores anônimos que contribuíram para a sua melhoria.

\title{
Segmentation model for water distribution networks based on the characteristics of consumer units
}

\begin{abstract}
In addition to incurring direct costs, the occurrence of breaks in a water distribution network always has associated social consequences, mainly related to the interruption of water supply during infrastructure repair. In these cases, network segmentation facilitates maintenance activities. However, the Brazilian norms, about network segmentation, relate only the technical guidelines that must be followed. We therefore propose network segmentation based on the impact intensity caused by a lack of water in each pipe segment. To measure this impact, we propose a priority index that aggregates the characteristics of consumer units by the SMARTER multicriteria method. Taking this index into account, we propose a segmentation model for water distribution networks that seeks to maximize the benefits generated and to minimize the number of isolation valves.
\end{abstract}

Keywords

Water distribution networks maintenance. Network segmentation. 1solation valves. Characteristics of consumer units. SMARTER. 